\title{
Nuclear magnetic resonance-paramagnetic relaxation enhancements: Influence of spatial quantization of the electron spin when the zero-field splitting energy is larger than the Zeeman energy
}

\author{
S. M. Abernathy, J. C. Miller, L. L. Lohr, and R. R. Sharp \\ Department of Chemistry, The University of Michigan, Ann Arbor, Michigan 48109
}

(Received 14 April 1998; accepted 27 May 1998)

\begin{abstract}
Dissolved paramagnetic ions generally provide an efficient mechanism for the relaxation of nuclear spins in solution, a phenomenon called the nuclear magnetic resonance-paramagnetic relaxation enhancement (NMR-PRE). Metal ions with electron spins $S \geqslant 1$ exhibit rich NMR relaxation phenomena originating in the properties of the zero-field splitting (zfs) interaction, which vanishes for spin- $-\frac{1}{2}$ ions but which is nonzero for $S \geqslant 1$ ions in site symmetry lower than cubic. For $S \geqslant 1$ ions in the vicinity of the zfs-limit, i.e., at magnetic-field strengths low enough that the zfs energy exceeds the Zeeman energy, the NMR-PRE depends strongly on the detailed structure of the electron spin energy levels as well as on the spatial quantization of the spin motion. It is shown theoretically and experimentally that the NMR-PRE produced by integer spins can be influenced strongly by the small intradoublet zero-field splittings, i.e., the splittings between the components of the non-Kramers doublets, which are produced by noncylindrical components of the crystal field potential. These small splittings produce relatively low-frequency oscillations in the dipolar field associated with $\left\langle S_{\hat{z}}\right\rangle$ (the spin component along the molecule-fixed $\hat{z}$ axis). These motions decouple the nuclear spin from the electron spin, thereby depressing, in some cases very strongly, the NMR-PRE. The presence of a relatively small Zeeman field, comparable in magnitude to the intradoublet spacing but small compared to the larger interdoublet zfs splittings, causes a major change in the spin wave functions which has profound effects on the motions of the electron spin. When the Zeeman energy exceeds the small zfs splitting, the oscillatory motion of $\left\langle S_{\hat{z}}\right\rangle$ damps out, with the result that the electron spin couples more effectively to the nuclear spin, providing a more efficient NMR relaxation pathway. NMR-PRE data are presented for the $S=1$ complex $\mathrm{Ni}(I I)(o \text {-pda })_{2} \mathrm{Cl}_{2}$ (o-pda=ortho-phenylenediamine) which confirm the importance of the splitting of the $m_{S}= \pm 1$ non-Kramers doublet on the NMR relaxation efficiency. The zfs $E$-parameter was measured from the NMR data to be $|E|=0.26 \mathrm{~cm}^{-1}$. The $S=2$ spin system, $\mathrm{Mn}(I I I)$-tetraphenylporphyrin sulfonate, exhibits a related phenomenon which arises from the effects of a small zfs splitting, $\Delta \epsilon_{ \pm 2}$, of the $m_{S}= \pm 2$ non-Kramers doublet that is caused by a fourfold rotational component of the crystal field potential. The splitting $\Delta \epsilon_{ \pm 2}$ was measured from NMR data to be $0.20 \mathrm{~cm}^{-1}$. (C) 1998 American Institute of Physics. [S0021-9606(98)01733-4]
\end{abstract}

\section{INTRODUCTION}

Dissolved paramagnetic ions generally produce large enhancements of the NMR relaxation rates of nuclear spins on solvent and ligand species. This phenomenon is called the NMR-paramagnetic relaxation enhancement or NMR-PRE. Ions with electron spins $S \geqslant 1$ exhibit very interesting NMR relaxation phenomena which originate in the properties of the zero field splitting (zfs) interaction. The zfs vanishes for spin- $\frac{1}{2}$ ions but is nonzero for $S \geqslant 1$ ions in site symmetry lower than cubic. For $S \geqslant 1$ ions, the NMR-PRE can conveniently be discussed with reference to the zfs- and Zeemanlimits, in which the zfs Hamiltonian, $H_{\mathrm{zfs}}$ is, respectively, much greater or much less than the Zeeman Hamiltonian, $H_{\text {Zeem }}$.

In a recent experimental study, two of us ${ }^{1}$ have shown that NMR relaxation enhancements produced by the high-spin $\quad S=1$ complex $\mathrm{Ni}(I I)(\mathrm{acac})_{2}\left(\mathrm{H}_{2} \mathrm{O}\right)_{2} \quad$ (acac $=$ acetylacetonato) in the vicinity of the zfs-limit are highly sensitive to the rhombic component of the quadratic zfs ten- sor (i.e., the component described by the electron spin resonance ESR $E$-parameter), much more so than to the cylindrical component (the $D$-parameter). To clarify the physical nature of the relaxation mechanism, the theory of electronnuclear dipole-dipole relaxation was subsequently cast ${ }^{2}$ in the molecular frame in a mathematical form which exhibits the dependence of the NMR relaxation efficiency on the spatial quantization of the electron spin and on the details of the electron spin motion. This analysis provides a highly intuitive physical picture of the magnetic dipole relaxation mechanism when $H_{\mathrm{zfs}}>H_{\text {Zeem }}$.

The present study explores in a more general context the relaxation mechanisms associated with the integer spin systems $S=1$ and $S=2$, with particular emphasis on the physical interpretation. Our objective is to better understand the relationship of the spin energy level diagram, the spatial quantization of the spin motion, and the NMR relaxation efficiency. It is shown that the NMR-PRE is particularly sensitive to the small level splittings within manifolds of non- 
Kramers doublets, splittings which are produced by noncylindrical components of the crystal field. Earlier work by Fukui et $a .^{3}$ and this laboratory ${ }^{1,2,4-6}$ have shown that the NMR-PRE for spin-1 is very sensitive to the presence of rhombic components in the zfs tensor. Related phenomena involving a dependence of the NMR-PRE on other noncylindrical components of the zfs tensor are of importance for other spin systems: It is shown below that for $S=2$, the NMR-PRE is particularly sensitive to fourth rank zfs terms which arise from components of the crystal field potential with fourfold rotational symmetry. Experimental examples are described in which both rhombic and fourth-order zfs splittings have been measured through the analysis of fielddependent NMR relaxation data.

The NMR-PRE is also sensitive, in a higher range of field strengths, to the large level splittings between adjacent non-Kramers or Kramers doublets. This latter dependence on interdoublet splittings has been described in some detail previously ${ }^{7,8}$ and is not further analyzed here. The main concern of the present work is the range of applied magneticfield strengths where the Zeeman energy is comparable to the small intradoublet zfs splittings but smaller than the interdoublet splittings. The NMR-PRE is generally very sensitive to the applied magnetic-field strength in this regime and provides experimental access to these relatively subtle features of the spin level system.

We also compare the nmr relaxation mechanisms associated with integer spins with those of half-integer $(S$ $\geqslant 3 / 2$ ) spins. The situation for half-integer spins is physically rather different from that for integer spins because of the unique physical properties of the central $m_{S}= \pm \frac{1}{2}$ Kramers doublet. The spatial quantization of the electron spin within the $m_{S}= \pm \frac{1}{2}$ manifold of an $S \geqslant 3 / 2$ ion in the vicinity of the zfs-limit differs from that of integer spins, a difference which is reflected in the NMR relaxation efficiency and in the molecular anisotropy of nmr relaxation. Differences characteristic of the low-field regime are analyzed.

New data are presented for the $S=1$ complex $\left[\right.$ trans $\left.-\mathrm{Ni}(I I)(o-\mathrm{pda})_{2} \mathrm{Cl}_{2}\right] \quad(o-\mathrm{pda}=$ ortho-phenylenediamine). The results confirm central aspects of the theory concerning the importance of zfs rhombicity for $S=1$ spin systems. We have also re-analyzed, using theory which accounts for the effects of zfs splittings, earlier experimental data $^{9}$ for the high-spin $S=2$ system, $\operatorname{Mn}(I I I)$-TPPS (TPPS $=$ tetraphenylporphyrin sulfonate). The NMR-PRE properties of $\mathrm{Mn}(I I I)$-TPPS and related $\mathrm{Mn}(I I I)$ porphyrins ${ }^{10}$ are highly unusual. The molar proton relaxivity of the axial water ligands is extremely large, larger per mole of bound water than that of the $\mathrm{Mn}^{2+}$ aquacation. Also, the water proton relaxation rate $\left(R_{1 p}\right)$ of $\mathrm{Mn}(I I I)$-porphyrins exhibits unique magnetic-field dependence in which $R_{1 p}$ increases markedly with increasing field strength in a remarkably low range of magnetic-field strengths, $0.04-0.4 \mathrm{~T}$. We have re-analyzed these data and show that the observed behavior is a consequence of the small intradoublet level splitting of the $m_{S}= \pm 2$ non-Kramers manifold, a splitting which arises from fourth-order terms of the crystal field potential. An analysis of the NMR-PRE data provides a sensitive measurement of this splitting.

\section{ZFS-LIMIT NMR RELAXATION THEORY}

We first summarize some results of the ZFS-limit NMRPRE theory of Ref. 2. This theory, which is appropriate to the vicinity of the zfs-limit where the total zfs energy is larger than the Zeeman energy, is cast in a molecular-frame description which provides a highly transparent physical interpretation of the spin relaxation mechanism. Related earlier work is described in Refs. 4-8, 11-15.

$R_{1 p}$ is the $T_{1}$ NMR rate of the paramagnetic solution after correction for the diamagnetic background of the solvent. Under slow motion conditions (see below), $R_{1 p}$ can be expressed as a sum of contributions due, respectively, to the Cartesian components, $\mu_{\hat{r}}(\hat{r}=\hat{x}, \hat{y}, \hat{z})$, of the electronic magnetic moment, $\mu=g_{e} \beta_{e} \mathbf{S}$ (which we assume is a spin-only quantity)

$$
\begin{aligned}
& R_{1 p}= R_{1 \hat{z}}+R_{1 \hat{x}}+R_{1 \hat{y}}, \\
& R_{1 \hat{r}}=(4 / 3)\left(\gamma_{I} g_{e} \beta_{e}\right)^{2}\left(\mu_{0} / 4 \pi\right)^{2} r_{I S}{ }^{-6}\left[1+P_{2}\left(\cos \theta_{\hat{r}}\right)\right] \\
& \times \sum_{\mu, \nu} P_{\mu}^{\circ}\left|\left\langle\mu\left|S_{\hat{r}}\right| \nu\right\rangle\right|^{2} J_{\hat{r}}\left(\omega_{\mu \nu}\right) \\
& J_{\hat{r}}(\omega)=\tau_{d} /\left(1+\omega^{2} \tau_{d}^{2}\right), \\
& 1 / \tau_{d}=1 / \tau_{S}^{(\mu)}+1 / \tau_{R}^{(1)} .
\end{aligned}
$$

Coordinates with a superscripting karat $\left(^{\wedge}\right)$ are defined in the molecule-fixed zfs-principal axis system. $S$ is the electron spin quantum number, $P_{2}(x)$ is the second-order Legendre polynomial, and $\theta_{\hat{r}}$ is the angle between the interspin vector $\mathbf{r}_{I S}$ and the $\hat{r}^{\text {th }}$ principal axis of the zfs-PAS. $\gamma_{I}$ is the nuclear magnetogyric ratio, $g_{e}=2.0023$ is the free electron $g$-value, $\beta_{e}$ is the Bohr magneton, and $\mu_{0}$ is permeability of free space. The sum is over the eigenstates, $|\mu\rangle,|\nu\rangle$, of the electron spin system. $P_{\mu}^{0}$ is the thermal equilibrium population of $|\mu\rangle$, assumed to be in the high-temperature limit, so that $P_{\mu}^{0}=(2 S+1)^{-1} \cdot \omega_{\mu \nu}$ is the $(\mu, \nu)$ transition frequency, and $\tau_{S}^{(\mu)}$ is the decay time of the time-correlation function $\left\langle\mu\left|S_{\hat{r}}(t) \cdot S_{\hat{r}}(0)\right| \mu\right\rangle . \tau_{d}$ is the correlation time of the $I-S$ dipolar interaction, and $\tau_{R}^{(1)}$ is the reorientational correlation time for a molecule-fixed vector. (It should be noted that the reorientational correlation time which appears in the traditional Zeeman-limit expressions for $R_{1 p}$ is that for a second rank molecule-fixed tensor, $\tau_{R}^{(2)}$. For molecules undergoing classical reorientational diffusion, $\left.\tau_{R}^{(1)}=3 \tau_{R}^{(2)}\right)$.

The physical picture underlying Eqs. (1)-(4) can be summarized, in brief, as follows. ${ }^{2}$ Each molecular frame cartesian component $R_{1 \hat{r}}$ in Eq. (1) can be interpreted as arising from the coupling of the nuclear spin magnetic moment with the local magnetic field arising from the molecular frame Cartesian component, $\left\langle\mu_{\hat{r}}\right\rangle$, of the electron spin's magnetic moment. The function $r_{I S}{ }^{-6}\left[1+P_{2}\left(\cos \theta_{\hat{r}}\right)\right]$ in Eq. (2) describes the spatial variation of the squared magnetic field of a magnetic dipole located at the origin and oriented along the $\hat{r}^{\text {th }}$ molecular axis. This local dipolar field is comprised of both static and oscillating components, the former proportional in amplitude to the diagonal matrix elements $\left|\left\langle\mu\left|\mu_{\hat{r}}\right| \mu\right\rangle\right|$, and the latter to the off-diagonal matrix elements, $\left|\left\langle\mu\left|\mu_{\hat{r}}\right| \nu\right\rangle\right|$. The off-diagonal fields oscillate at the associated transition frequencies, $\omega_{\mu \nu}$. The spectral density 
functions $J_{\hat{r}}\left(\omega_{\mu \nu}\right)$ describe the dipolar power density produced by $\left|\left\langle\mu\left|\mu_{\hat{r}}\right| \nu\right\rangle\right|$ at the nuclear Larmor frequency $\omega_{I}$ (we assume $\omega_{\mu \nu} \gg \omega_{I}$ ). The terms in Eq. (2) for which the transition frequency $\omega_{\mu \nu}$ is large enough that $\omega_{\mu \nu} \tau_{d} \gg 1$ produce very little power density at $\omega_{I}$ and, therefore, contribute negligibly to $R_{1 p}$.

Two important approximations underlying Eqs. (1)-(4) should be noted. First, the electron spin relaxation times $\tau_{S}^{(\mu)}$ are in general magnetic-field dependent, but unfortunately we have at present no analytic theory of this dependence that is valid outside the Zeeman limit. Electron spin relaxation of $S \geqslant 1$ ions is usually assumed to result physically from the stochastic fluctuations of the zfs-tensor arising from collisional distortions and molecular reorientation of the metal coordination sphere. In the Zeeman-limit, the theory of Bloembergen and Morgan ${ }^{16}$ (B-M) provides an appropriate description

$$
\begin{aligned}
\tau_{S}^{-1}= & \left(2 \Delta_{t}^{2} / 50\right)[4 S(S+1)-3] \\
& \times\left(\frac{\tau_{v}}{1+\left(\omega_{S} \tau_{v}\right)^{2}}+\frac{4 \tau_{v}}{1+\left(2 \omega_{S} \tau_{v}\right)^{2}}\right) .
\end{aligned}
$$

$\Delta_{t}$ is the rms amplitude of the time-dependent part of the zfs tensor, $\tau_{v}$ is a correlation time for the zfs fluctuation, and $\omega_{S}$ is the electron Larmor frequency. However, the Larmor frequencies $\omega_{S}$ do not realistically (or even approximately) describe the spin level splittings when $H_{\mathrm{zfs}}>H_{\text {Zeem }}$, and thus $\mathrm{B}-\mathrm{M}$ theory is not appropriate in this regime. Also, B-M theory does not account for magnetic-field dependence of the spin wave functions, which can be very large when the zfs splittings are comparable to the Zeeman energy (see below). Westlund ${ }^{17}$ has recently developed a molecular frame zfslimit Redfield theory which describes $\tau_{S}$ of $S=1$ ions under slow motion conditions. However, this theory does not incorporate the effects of field variation either in the spin matrix elements or in the transition frequencies and thus is a zerofield limiting theory; i.e., it does not describe the magneticfield dependence of $\tau_{S}$. A suitable analytical theory is needed.

In the experimental analyses described below, attention is focused on the relationship between the NMR-PRE and the coherent oscillational motions of the electron spin, and the magnetic-field dependence of $\tau_{S}$ is neglected. The coherent electron spin dynamics driven by the static spin Hamiltonian are strongly magnetic-field dependent due to the magnetic-field dependence of the spin eigenfunctions in the low field regime (see below). It is shown below that the slow motion theory is strikingly successful in describing the fielddependence of NMR-PRE data even when the $\tau_{S}$ parameters are assumed to be field-independent quantities. The degree to which magnetic-field dependence in $\tau_{S}$ may produce additional field dependence in $R_{1 p}$ needs further study.

An additional approximation underlying Eqs. (1)-(5) is an incomplete description of the effects of Brownian reorientation. Although reorientational effects are included in part within the definition of $\tau_{d}$, which describes temporal variations in the electron-nuclear dipolar coupling, fluctuations in the electron spin Hamiltonian are not described: molecular tumbling produces time dependence in the transition fre- quencies $\omega_{\mu \nu}$ and in the matrix elements $\left\langle\mu\left|S_{\hat{r}}\right| \nu\right\rangle$ of Eq. (2), due to the fact that $H_{\mathrm{zfs}}$ is explicitly time dependent, while the analytic theory treats these quantities as static entities as they would be in a powder. Reorientational modulation of $H_{S}$ can also provide a contributing relaxation pathway to $\tau_{S}^{(\mu)}$. The neglect of these effects is valid strictly when the reorientational correlation time is much longer than the electron spin relaxation time.

More general computational approaches which account accurately for the effects of Brownian reorientation as well as for the effects of comparable Zeeman and zfs terms in the spin Hamiltonian are described in Refs. 16-27. The most recent and perhaps the most powerful of these are methods based on spin dynamics simulation, which have been developed both in this laboratory ${ }^{25}$ and in Sweden. ${ }^{26,27}$ However, the zfs-limit analytic theory of Ref. 2 is, in our view, of much greater utility than the computational approaches in that the latter obscure important physical aspects of the relaxation mechanism which are transparent in the former. This not only provides greater physical insight into the relaxation process but also provides a much clearer understanding of the information content of the NMR-PRE experiment. For this reason, we prefer to use the analytic theory of Ref. 2 for the primary analysis of data and to use the spin dynamics program SPINDYN. $\mathrm{f}^{25}$ for refining the analysis and assessing its accuracy.

\section{SPIN WAVE FUNCTIONS AND LEVEL DIAGRAMS}

In this and the following sections we discuss the dependence of $R_{1 p}$ on the spatial quantization of the electron spin motion as determined by the spin eigenfunctions and level splittings. It is shown in particular that the motions of the time correlation functions $\left\langle S_{\hat{r}}(t) \cdot S_{\hat{r}}(0)\right\rangle$ are highly sensitive at relatively low magnetic-field strengths to the small zfs splittings within non-Kramers doublets and that this dependence has profound consequences for NMR relaxation phenomena. At higher field strengths, the spin motion is also sensitive to the larger interdoublet splittings. The zfs level structure depends on the detailed form and symmetry of the crystal field potential. These factors are analyzed systematically in this and the following sections. The results are then applied to the interpretation of experimental data for two model integer spin systems, one containing the $S=1 \mathrm{Ni}(I I)$ ion, the other containing high spin $\operatorname{Mn}(I I I)$, an $S=2$ ion.

$d$-Block ions with quenched orbital angular momentum have spin level systems which can be described by a spin Hamiltonian of the form,

$$
\begin{aligned}
H_{S}= & B_{0}^{(2)} O_{0}^{(2)}+B_{2}^{(2)} O_{2}^{(2)} \\
& +B_{0}^{(4)} O_{0}^{(4)}+B_{2}^{(4)} O_{2}^{(4)}+B_{4}^{(4)} O_{4}^{(4)}+g_{e} \beta_{e} \mathbf{B}_{o} \cdot \mathbf{S} .
\end{aligned}
$$

The last term on the rhs is the electronic Zeeman Hamiltonian. The quantities $O_{q}^{(k)}$ of rank $k$ and degree $q$ are functions of the spin operators which transform like the functions $\cos (q \varphi)$ under rotations about the molecular $\hat{z}$ axis. The functions for $k=2,4$ are given in the Appendix. Table 16 of Abragram and Bleaney ${ }^{28}$ contains a more extensive compilation but with different notation: Their $O_{k}^{q}$ is written $O_{q}^{(k)}$ in Eq. (6). Matrix elements $\left\langle S, m_{S}\left|O_{q}^{(k)}\right| S, m_{S}^{\prime}\right\rangle$ vanish for all $k$ 


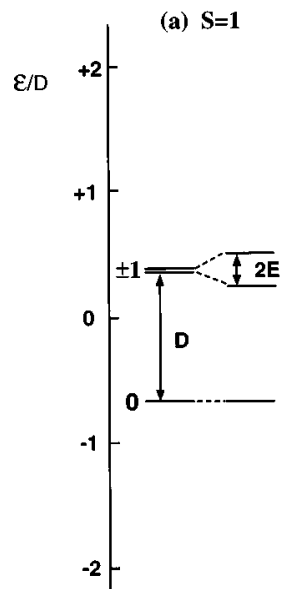

(b) $\mathrm{S}=\mathbf{3 / 2}$

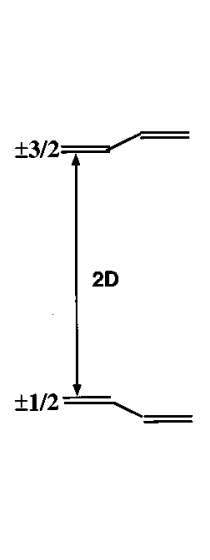

(c) $\mathrm{S}=2$

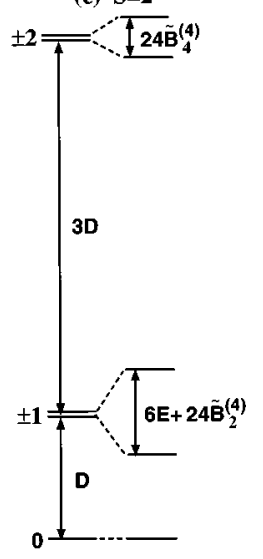

FIG. 1. Zfs-limit spin level diagrams for (a) $S=1$, (b) $S=\frac{3}{2}$, and (c) $S=2$ in cylindrical (left) and orthorhombic (right) crystal field site symmetry. $\widetilde{B}_{j}^{(k)}$ in $\mathrm{cm}^{-1}$.

$>2 S$. Thus sixth and higher rank terms are absent for $S<3$ and are consequently unimportant for $d$-block ions; they will not be considered further here, although they can be quite important for lanthanides. Matrix elements $\left\langle S, m_{S}\left|O_{q}^{(k)}\right| S, m_{S}^{\prime}\right\rangle$ also vanish for $q \neq\left|m_{S}-m_{S}^{\prime}\right|$. The coefficients $B_{q}^{(k)}$ are parameters which describe the zfs tensor in the principal axis system. The second rank terms $(k=2)$ in Eq. (6) are related to the zfs parameters $D$ and $E$ of ESR spectroscopy: $B_{0}^{(2)}=h c\left(10^{2}\right)(D / 3)$ and $B_{2}^{(2)}=h c\left(10^{2}\right) E$, where $D$ and $E$ are in units of $\mathrm{cm}^{-1}$, and Planck's constant $h$ and the speed of light $c$ are in SI. The $B_{q}^{(k)}$ are usually calculated by perturbation theory in terms of the matrix elements of the spin-orbit coupling Hamiltonian between the ground and excited electronic states. The $B_{q}^{(k)}$ depend on the strength and symmetry of the crystal field (CF) potential. Terms $q=0$ arise from cylindrical CF components (i.e., those independent of $\varphi$ ), $q=2$ terms from CF components with twofold rotational symmetry, $q=4$ terms from CF components with fourfold rotational symmetry, etc.

Spin level diagrams in the zfs-limit for cylindrical and orthorhombic CF symmetry are shown in Fig. 1 for $S=1, \frac{3}{2}$, and 2 . We are primarily concerned with the physical situation for integer spins in the vicinity of the zfs-limit $\left(H_{\mathrm{zfs}}\right.$ $\left.>H_{\text {Zeem }}\right)$. We use a perturbation analysis in which the cylindrical zfs terms of Eq. (6) define the zero-order Hamiltonian, to which the Zeeman and noncylindrical zfs terms are treated as perturbations. For $S=1$, zfs terms higher than quadratic $(k=2)$ vanish. In a $\mathrm{CF}$ potential with threefold or higher rotational symmetry, the $m_{S}= \pm 1$ non-Kramers doublet levels are degenerate and $m_{S}$ is a good quantum number. The spin eigenfunctions can be chosen as the circularly polarized functions, $\{| \pm 1\rangle,|0\rangle\}$, quantized along the molecular $\hat{z}$ axis (these functions are identical to the Zeeman basis functions when the external magnetic field is parallel to the molecular $\hat{z}$ axis). The presence of a rhombic term $B_{2}^{(2)}$ arising from a twofold component in the $\mathrm{CF}$ gives rise to nonzero matrix elements between the $m_{S}=+1$ and $m_{S}=-1$ eigenstates and thus breaks the degeneracy of the $m_{S}= \pm 1$ non-Kramers doublet in first order (Fig. 1) ('first order'" refers to a con- tribution to the energy that is linear in the coefficient $B_{2}^{(2)}$ ). In a rhombic field, $m_{S}$ is no longer a good quantum number, and the eigenstates are Cartesian-polarized linear combinations of circularly polarized functions

$$
\begin{aligned}
& \left|0^{\prime}\right\rangle=|S, 0\rangle, \\
& \left| \pm 1^{\prime}\right\rangle=2^{-1 / 2}(-|S,+1\rangle \pm|S,-1\rangle) .
\end{aligned}
$$

These functions, $\left\{\left|+1^{\prime}\right\rangle,\left|-1^{\prime}\right\rangle,\left|0^{\prime}\right\rangle\right\}$, transform spatially as the rotations $\left\{R_{x}, R_{y}, R_{z}\right\}$ and can be relabeled $\{|X\rangle,|Y\rangle,|Z\rangle\}$.

For $S=2$, zfs terms of rank $k=2$ and 4 will in general be present, depending on the symmetry of the CF. The $B_{0}^{(2)}$ cylindrical term splits the five spin levels into a singlet and two non-Kramers doublets $\left(m_{S}=0, \pm 1\right.$, \pm 2$)$ with energies $\epsilon_{0}^{(2)}=B_{0}^{(2)}\left(3 m_{S}^{2}-6\right)$. The $B_{0}^{(4)}$ term modifies these energies by an additional amount $\epsilon_{0}^{(4)}=B_{0}^{(4)}\left(35 m_{S}^{4}-95 m_{S}^{2}+72\right)$ but does not split the doublets. $B_{0}^{(4)}$ has no important qualitative effects on $R_{1 p}$ beyond those produced by $B_{0}^{(2)}$, and we do not consider it further. The spin eigenfunctions in a cylindrical $\mathrm{CF}$ can be chosen as the circularly polarized wave functions, $\{|0\rangle,| \pm 1\rangle,| \pm 2\rangle\}$, spatially quantized along $\hat{z}$. The rhombic zfs terms $B_{2}^{(2)}$ and $B_{2}^{(4)}$ couple levels for which $\Delta m_{S}= \pm 2$ and thus produce a first-order splitting of the degenerate $m_{S}= \pm 1$ manifold of $S=2$. Zfs rhombicity does not split the $m_{S}= \pm 2$ manifold in first order. The term containing $B_{4}^{(4)}$ in Eq. (6) arises from a fourfold symmetry component in the $\mathrm{CF}$. This term couples levels with $\Delta m_{S}= \pm 4$ and thus splits the $m_{S}= \pm 2$ Kramers doublet in first-order perturbation theory (Fig. 1). The effects of $B_{4}^{(4)}$ are likely to be quite important for $S=2$ ions, as is described below with reference to $\operatorname{Mn}(I I I)$-TPPS.

The wave functions for $S=2$ in a noncylindrical CF site symmetry are given by Eqs. (7) plus the additional Cartesian function

$$
\left| \pm 2^{\prime}\right\rangle \equiv 2^{-1 / 2}(|S,+2\rangle \pm|S,-2\rangle) .
$$

The Cartesian basis functions for $S=2$ transform spatially like the $d$-orbitals and can be labeled $\left\{\left|Z^{2}\right\rangle,|X Z\rangle,|Y Z\rangle, \mid X^{2}\right.$ $\left.\left.-Y^{2}\right\rangle,|X Y\rangle\right\}$ for $\left\{|0\rangle,\left| \pm 1^{\prime}\right\rangle,\left| \pm 2^{\prime}\right\rangle\right\}$, respectively.

\section{SPATIAL QUANTIZATION AND NMR RELAXATION EFFICIENCY}

It can be seen from Eq. (2) that the NMR relaxation efficiency and its molecular anisotropy are closely related to the spatial quantization and to the motional characteristics of $\langle S\rangle$, as described by the time correlation functions

$$
\sum_{\mu}\left\langle\mu\left|S_{\hat{r}}(0) \cdot S_{\hat{r}}(t)\right| \mu\right\rangle=\sum_{\mu, \nu}\left|\left\langle\mu\left|S_{\hat{r}}\right| \nu\right\rangle\right|^{2} \exp \left(-i \omega_{\mu \nu} t\right),
$$

where $\{|\mu\rangle,|\nu\rangle\}$ are the eigenbasis of the static spin Hamiltonian, $H_{S}$. Diagonal matrix elements, $\left\langle\mu\left|S_{\hat{r}}\right| \mu\right\rangle$ (when these do not vanish by symmetry) describe zero-frequency spin motions. The associated spectral density functions, $J_{\hat{r}}(0)$ in Eq. (2), are relatively large. These terms, when present, provide relatively efficient relaxation pathways for nuclear spins. Off-diagonal matrix elements, $\left\langle\mu\left|S_{\hat{r}}\right| \nu\right\rangle$, describe oscillations of $\left\langle S_{\hat{r}}\right\rangle$ at the transition frequency $\omega_{\mu \nu}$. 
The dipolar spectral density functions, $J\left(\omega_{\mu \nu}\right)$, associated with these terms are relatively small when the level splitting is large enough that $\omega_{\mu \nu} \tau_{d}>1$, in which case the electron spin is motionally decoupled from the nuclear spin due to the effects of spin oscillation. As an example, for $\omega_{\mu \nu}$ $=2 \mathrm{~cm}^{-1}$, this condition is met for $\tau_{d}>2.6 \mathrm{ps}$. Thus interdoublet zfs splittings the order of a few wave numbers can usually be neglected as relaxation pathways unless the electron spin relaxation time is extremely short, at most the order of a couple of ps. Thus the principal relaxation terms in Eq. (2) will usually be those containing diagonal matrix elements (when these are nonvanishing) as well as terms arising from small intradoublet splittings.

We consider first the situation for an integer spin when the spin Hamiltonian is the zero order Hamiltonian, i.e., when the spin is in the zfs-limit $\left(B_{o}=0\right)$ in cylindrical CF site symmetry. The spin eigenfunctions are the circularly polarized functions $\left|S, m_{S}\right\rangle$ quantized along the molecular $\hat{z}$ axis. $\left\langle S_{\hat{z}}\right\rangle= \pm m_{S}$ is a constant of the motion, and $\left\langle S_{\hat{x}}\right\rangle$ and $\left\langle S_{\hat{y}}\right\rangle$ oscillate at the $1 Q$ frequencies, $\omega_{D}$ for $S=1$ and $\left(\omega_{D}, 3 \omega_{D}\right)$ for $S=2$ (the level systems are shown in Fig. 1). Ignoring matrix elements which describe spin oscillations with frequencies the order of $n \omega_{D}$, the principal NMR relaxation pathway will result only from terms in $\left\langle S_{\hat{z}}\right\rangle$, and the relative relaxation efficiencies of the different eigenstates are proportional to $m_{S}^{2}$. For $S=1$, the largest part of the NMR relaxation efficiency is provided by $\left\langle S_{\hat{z}}\right\rangle$ in the $| \pm 1\rangle$ eigenstates. For $S=2$, the relaxation efficiency is provided by the $| \pm 1\rangle$ and $| \pm 2\rangle$ eigenstates, which contribute in the ratio 1:4. The molecular anisotropy of $R_{1 p}$ is that of $R_{1 \hat{z}}$, as given by the function $1+P_{2}\left(\cos \theta_{\bar{z}}\right)$.

If an orthorhombic component $\left(B_{2}^{(2)} \neq 0, B_{2}^{(4)} \neq 0\right)$ is present in addition to the cylindrical components of the $\mathrm{CF}$ potential, the eigenfunctions at zero field are the Cartesianpolarized functions of Eq. (7). In the eigenbasis of $H_{S}$, the diagonal matrix elements of $\left\langle S_{\hat{z}}\right\rangle$ then vanish, $\left\langle 0\left|S_{\hat{z}}\right| 0\right\rangle$ $=\left\langle \pm 1^{\prime}\left|S_{\hat{z}}\right| \pm 1^{\prime}\right\rangle=\left\langle \pm 2^{\prime}\left|S_{\hat{z}}\right| \pm 2^{\prime}\right\rangle=0$, while the offdiagonal matrix elements, $\left\langle \pm 1^{\prime}\left|S_{\hat{z}}\right| \mp 1^{\prime}\right\rangle,\left\langle \pm 2^{\prime}\left|S_{\hat{z}}\right| \mp 2^{\prime}\right\rangle$, which couple the levels within the non-Kramers doublets are large. Terms containing the off-diagonal matrix elements oscillate at the small transition frequencies of the intradoublet splittings, which are $\Delta \omega_{ \pm 1}=S(S+1) \omega_{E}$, and $\Delta \omega_{ \pm 2}$ $=24 \hbar^{-1} B_{4}^{(4)}$ in first order for the $m_{S}= \pm 1,2$ non-Kramers doublets, respectively. The resulting motion in $\left\langle S_{\hat{z}}\right\rangle$ acts to suppress the NMR-PRE. Experimental examples for $S=1$ and $S=2$ are discussed in the following sections.

We now consider the magnetic-field dependence of $R_{1 p}$ in the vicinity of the orthorhombic zfs-limit: it is assumed that a Zeeman interaction is present which is small compared to the large interdoublet splittings between non-Kramers doublets but which may be comparable to or larger than the small intradoublet splittings. The first-order energies in the presence of the Zeeman field and the noncylindrical zfs field are

$$
\begin{aligned}
& \epsilon_{0}=-6 B_{0}^{(2)} \\
& \epsilon_{ \pm 1}=-3 B_{0}^{(2)} \pm\left[\left(\Delta \epsilon_{ \pm 1}^{\circ}\right)^{2}+z^{2}\right]^{1 / 2}, \\
& \epsilon_{ \pm 2}=+6 B_{0}^{(2)} \pm\left[\left(\Delta \epsilon_{ \pm 2}^{\circ}\right)^{2}+(2 z)^{2}\right]^{1 / 2}
\end{aligned}
$$

where

$$
\begin{aligned}
& \Delta \epsilon_{ \pm 1}^{\circ}=B_{2}^{(2)} S(S+1) / 2, \\
& \Delta \epsilon_{ \pm 2}^{\circ}=12 B_{4}^{(4)}, \\
& Z=g_{e} \beta_{e} B_{o} \cos (\theta) .
\end{aligned}
$$

$\theta$ is the angle between $\mathbf{B}_{\mathbf{0}}$ and $\hat{z} .2 \Delta \boldsymbol{\epsilon}_{ \pm 1}^{\circ}$ and $2 \Delta \boldsymbol{\epsilon}_{ \pm 2}^{\circ}$ are the first-order level splittings of the $\Delta m_{S}= \pm 1$ and \pm 2 nonKramers doublets in the absence of a Zeeman field [Eqs. (9c) and (9e) apply to $S=2$ but not $S=1$ ]. Only the component of $\mathbf{B}_{\mathbf{0}}$ parallel to $\hat{z}$ contributes to the Zeeman energy in first order.

The presence of a Zeeman interaction comparable in magnitude to the small zfs splittings causes major changes in the spin wave functions. In the limit $B_{o} \rightarrow 0$, the eigenfunctions are the Cartesian-polarized functions of Eq. (7). When $Z$ is greater than the intradoublet zfs splitting (but smaller than the interdoublet splittings), these eigenfunctions uncouple and approach the circularly polarized functions which are spatially quantized along $\hat{z}$. As long as the Zeeman energy is smaller than the large interdoublet zfs splittings ( $Z$ $\left\langle n \hbar \omega_{D}\right),\langle\mathbf{S}\rangle$ remains spatially quantized along molecular, rather than laboratory axes. The principal effect of $B_{o}$ is to uncouple the eigenfunctions of Eq. (7), thereby damping out the oscillations in $\left\langle S_{\hat{z}}\right\rangle$. As a result, the NMR relaxation efficiency rises dramatically. When the Zeeman energy rises further, exceeding the large zfs splittings, the eigenfunctions change again, this time from the circularly polarized functions quantized along $\hat{z}$ to the circularly polarized Zeeman functions quantized along laboratory $z$ axis. The changes in NMR relaxation efficiency which accompany this latter change in spatial quantization have been described previously. ${ }^{7-9}$

The changes in the wave functions and energies which occur for $S=1$ when $B_{o}$ rises from zero to a value larger than the rhombic splitting $2 B_{2}^{(2)}$ are shown in Figs. 2(a) and 2(b). The first-order eigenfunctions of the $m_{S}= \pm 1$ nonKramers doublet can be written

$$
\begin{aligned}
& \left|\Psi_{+1}\right\rangle=c_{1}|S,+1\rangle+c_{2}|S,-1\rangle, \\
& \left|\Psi_{-1}\right\rangle=-c_{2}|S,+1\rangle+c_{1}|S,-1\rangle .
\end{aligned}
$$

Figure 2(a) shows the coefficients $\left|c_{1}\right|$ and $\left|c_{2}\right|$ as a function of $\left(g_{e} \beta_{e} B_{o} / 2 B_{2}^{(2)}\right)$ at $\theta=\pi / 4$ (the angle between $z$ and $\hat{z}$ ). When $B_{o}=0,\left|c_{1}\right|=\left|c_{2}\right|=2^{-1 / 2}$, and the eigenfunctions are identical to the $\left| \pm 1^{\prime}\right\rangle$ coupled eigenfunctions of Eq. (7). With increasing $B_{o}$, the $\left|c_{2} / c_{1}\right|$ ratio decreases, approaching 0 in the limit $\left(g_{e} \beta_{e} B_{o} / 2 B_{2}^{(2)}\right) \gg 1$. The matrix elements of $\left\langle S_{\hat{z}}\right\rangle$ change accordingly: the diagonal matrix elements of $\left\langle S_{\hat{z}}\right\rangle$ rise from zero at $B_{o}=0$ toward values of $\left\langle S_{\hat{z}}\right\rangle=m_{S}$ when $\left(\omega_{S} / 2 \omega_{E}\right) \gg 1$, while the off-diagonal matrix elements of $\left\langle S_{\hat{z}}\right\rangle$ decrease in a mirror fashion. Thus the oscillation of $\left\langle S_{\hat{z}}\right\rangle$ that is driven by the rhombic splitting is damped out by the effects of the Zeeman interaction. A useful means of visualizing the effects of these changes on $R_{1 p}$ is through plots of the dipolar power spectrum, as we have shown previously $^{2}$ for $S=1$. A further example is given below in regard to the $S=2$ complex, Mn-TPPS. 

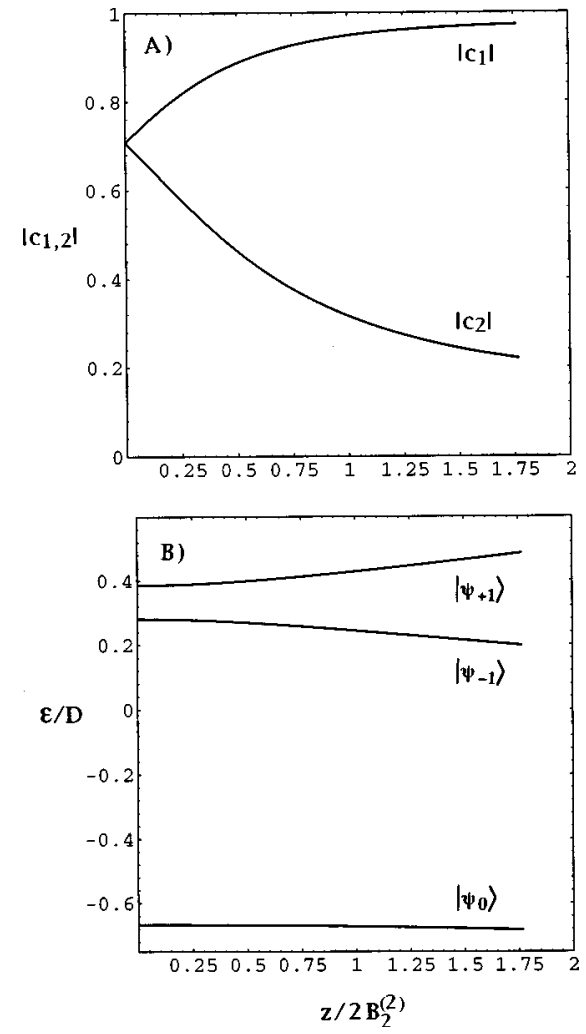

FIG. 2. Change of the spin eigenfunctions (a) and energies (b) of the $m_{S}$ $= \pm 1$ non-Kramers manifold with increasing Zeeman field strength for a spin $S=1$ with a rhombic zfs tensor. The coefficients $c_{1,2}$ are defined in Eq. (8) of the text. Calculations assume an angle $\theta=\pi / 4$ between the laboratory $z$ and molecular $\hat{z}$ axis.

Analogous phenomena occur for the $m_{S}= \pm 2$ doublet of $S=2$, although in this case the change in wave function occurs when the Zeeman energy rises above the splitting of the $m_{S}= \pm 2$ levels, which is produced in first order by the fourth rank zfs term, $24 B_{4}^{(4)}$, and in second order by the rhombic term, $B_{2}^{(2)}$. The first-order eigenfunctions of the $m_{S}= \pm 2$ manifold in the presence of the Zeeman field are the coupled functions

$$
\begin{aligned}
& \left|\Psi_{+2}\right\rangle=c_{1}^{\prime}|S,+2\rangle+c_{2}^{\prime}|S,-2\rangle, \\
& \left|\Psi_{-2}\right\rangle=-c_{2}^{\prime}|S,+2\rangle+c_{1}^{\prime}|S,-2\rangle .
\end{aligned}
$$

A Zeeman energy greater than $\Delta \epsilon_{ \pm 2}^{\circ}$ uncouples the circularly polarized functions in a manner analogous to that described above for $S=1$ subject to a rhombic zfs interaction. The effect of the $B_{4}^{(4)}$ term is likely to be quite important in practice for $S=2$ because of the large potential NMR relaxation efficiency of the $m_{S}= \pm 2$ eigenstates $\left(R_{1 p}\right.$ varies as $\left|m_{S}\right|^{2}$ when the oscillations of $\left\langle S_{\hat{z}}\right\rangle$ are damped out by $B_{o}$ ). Also, the splitting due to $B_{4}^{(4)}$ is likely to be smaller than typical rhombic splittings unless the latter vanish by symmetry, resulting in a relatively small oscillation frequency of $\left\langle S_{\hat{z}}\right\rangle$ and a correspondingly high relaxation efficiency.

\section{HALF-INTEGER SPINS: $S=3 / 2$}

The situation for half-integer spins is physically rather different from that for integer spins, in part because of the unique properties of the $m_{S}= \pm \frac{1}{2}$ Kramers doublet. For $S$ $=\frac{3}{2}$ in the cylindrical zfs-limit, the $m_{S}= \pm \frac{3}{2}$ and $m_{S}= \pm \frac{1}{2}$ Kramers doublets are split by $2 D$ (Fig. 1). The Kramers doublets are not split by a CF potential of any symmetry or rank. A rhombic zfs $E$ term produces only a second-order contribution to the cylindrical splitting. The eigenfunctions in a rhombic $\mathrm{CF}$ can be chosen (in zero field) as the circularly polarized functions $\left|S, m_{S}\right\rangle$, quantized along the molecular $\hat{z}$ axis (although any linear combination of these functions may equally well be selected within the degenerate manifold).

The Kramers doublets split in the presence of the applied magnetic field. When a Zeeman energy smaller than the interdoublet splitting is applied, the spatial quantization differs for the $m_{S}= \pm \frac{1}{2}$ and $m_{S}= \pm \frac{3}{2}$ doublets. The $\pm \frac{3}{2}$ wave functions are not mixed by the magnetic field in first order, and their first-order energies are

$$
\epsilon_{ \pm 3 / 2}=D \pm(3 / 2) g_{e} \beta_{e} B_{o} \cos (\theta) .
$$

The spin eigenfunctions are the circularly polarized functions $\left|S, \pm \frac{3}{2}\right\rangle$, quantized along the molecular $\hat{z}$ axis. $\left\langle S_{\hat{z}}\right\rangle= \pm \frac{3}{2}$ is a constant of the motion, while $\left\langle S_{\hat{x}}\right\rangle$ and $\left\langle S_{\hat{y}}\right\rangle$ oscillate at the $1 Q$ frequency, $2 \omega_{D}$. Thus the situation for the $m_{S}= \pm \frac{3}{2}$ manifold with respect to NMR relaxation efficiency is similar to that for integer spins in that $R_{1 p}$ results principally from $\left\langle S_{\hat{z}}\right\rangle$, and the molecular anisotropy of $R_{1 \hat{z}}$ is given by the function $\left[1+P_{2}\left(\cos \theta_{\hat{z}}\right)\right]$.

The situation is rather different for the $m_{S}= \pm \frac{1}{2}$ doublet, since $B_{o}$ mixes the doublet eigenstates in first order. The eigenfunctions must be found by re-diagonalizing $H_{S}$ within the $m_{S}= \pm \frac{1}{2}$ manifold. The eigenfunctions are circularly polarized Zeeman functions, $\left|S, \pm \frac{1}{2}\right\rangle$, quantized along the external field rather than along molecular axes. The first-order energies are

$$
\epsilon_{ \pm 1 / 2}=-D \pm\left(\frac{1}{2}\right) g(\theta) \beta_{e} B_{o} .
$$

where $g(\theta)$ is the anisotropic $g$-value. Because of this requantization from molecular to laboratory axes, the $m_{S}$ $= \pm \frac{1}{2}$ doublet has NMR relaxation properties more similar to those of a spin $\frac{1}{2}$ radical than to the integer spin systems described above, although the $g$-tensor within the $\pm \frac{1}{2}$ manifold is anisotropic and has a trace that differs from the free electron $g$-value. When referenced to a fictitious spin- $\frac{1}{2}$ system, the $g$-values for the $m_{S}= \pm \frac{1}{2}$ Kramers doublet of $S=\frac{3}{2}$ are $g_{\|}=g_{e}$, and $g_{\perp}=2 g_{e}$, so that $\langle g\rangle=\left(\frac{5}{3}\right) g_{e} \cdot\left\langle S_{z}\right\rangle$ (the component along the laboratory $z$ axis), is a constant of the motion equal to $\pm \frac{1}{2}$ and thus contributes strongly to the relaxation efficiency. $\left\langle S_{x}\right\rangle$ and $\left\langle S_{y}\right\rangle$ precess at an average Larmor frequency $\left\langle\omega_{L}\right\rangle=\langle g\rangle \beta_{e} B_{o}$. These components may also contribute significantly to the relaxation efficiency when $\left\langle\omega_{L}\right\rangle \tau_{d} \leqslant 1$.

\section{$S=1:\left[\right.$ trans $\left.-\mathrm{Ni}(I I) \mathrm{Cl}_{2}(0-\text { phenylenediamine })_{2}\right]$}

The phenomena described above for $S=1$ have been documented experimentally for the complex $\left[\mathrm{Ni}(I I)(\operatorname{acac})_{2}\left(\mathrm{H}_{2} \mathrm{O}\right)_{2}\right]$ in previous studies. ${ }^{1,2}$ In this section, new data are presented for the $S=1$ complex $\mathrm{Ni}(o \text {-pda })_{2} \mathrm{Cl}_{2}$ $\left[\right.$ trans $\left.-\mathrm{Ni}(I I) \mathrm{Cl}_{2}(o \text {-phenylenediamine })_{2}\right]$ which further il- 


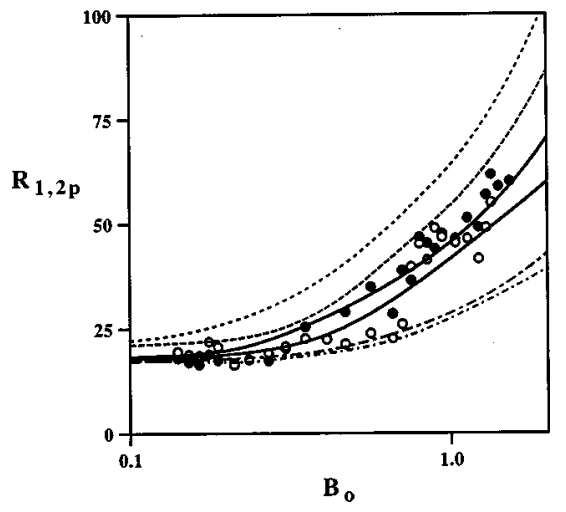

FIG. 3. Magnetic-field dependence of the NMR relaxation enhancements of the $H_{4,5}$ aromatic proton resonance of $\mathrm{Ni}(I I) \mathrm{Cl}_{2}(o-\mathrm{dpa})_{2}$ at $20^{\circ} \mathrm{C}$. Data for $R_{1 p}$ are open circles, $R_{2 p}$ are filled circles. Curves show results of theoretical simulations based on Eq. (2) of the text. $R_{1 p}$ is the upper and $R_{2 p}$ the lower of each pair of curves. Assumed parameters are: (dashes) $\tau_{S}$ $=30 \mathrm{ps}, E=0.213 \mathrm{~cm}^{-1}$; (solid lines) $\tau_{S}=18 \mathrm{ps}, E=0.263 \mathrm{~cm}^{-1}$; (dotdash) $\tau_{S}=9 \mathrm{ps}, E=0.326 \mathrm{~cm}^{-1}$. Simulations assumed $D=5 \mathrm{~cm}^{-1}, \tau_{R}^{(1)}$ $=432 \mathrm{ps}$, but were nearly independent of these parameters.

lustrate the nature of the effect and confirm the generality of its importance for $S=1$ in orthorhombic site symmetry.

Figure 3 shows the magnetic-field dependence of the NMR relaxation enhancements $\left(R_{1 p}\right.$ and $\left.R_{2 p}\right)$ of the aromatic proton resonances of $\mathrm{Ni}\left(o\right.$-pda) ${ }_{2} \mathrm{Cl}_{2}$ (Fig. 4$)$ in DMSO solution across a range of magnetic-field strengths from 0.05 to $1.8 \mathrm{~T}$. Measurements were carried out using using solutions in dimethylsulfoxide (DMSO) $-d_{6}$, so that the resonance observed was that of the aryl protons of the solute. The x-ray crystal structure ${ }^{29}$ shows that the inequivalent protons in the $(3,6)$ and $(4,5)$ positions are 4.50 and $5.93 \AA$, respectively, from the metal ion, giving a ratio $r^{-6}(3,6) / r^{-6}(4,5) \cong 5.3$. The relaxation decays were analyzed by ignoring an initial segment of the decay equal to approximately 1.6 times the shorter $T_{1}$. Under these conditions, the measured $R_{1}$ is a weighted quantity reflecting predominantly the decay of the $(4,5)$ protons. The decay appeared mono-exponential within the scatter in the data (which was, however, substantial). In the data analysis, an effective distance of $5.5 \AA$ was used. This seems a reasonable approximation for our purpose, which is to investigate the mechanistic questions described above, particularly whether changes in the spatial quantization of $\left| \pm 1^{\prime}\right\rangle$ spin states underlies the observed magnetic-field dependence of the $R_{1,2 p}$ field dispersion profiles.

$R_{1 p}$ approximately equalled $R_{2 p}$ across the range of magnetic-field strengths investigated, although because of the scatter in the data, a systematic difference the order of

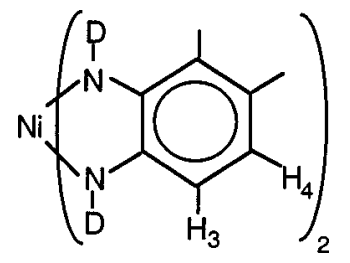

FIG. 4. Numbering of the ligand protons in $\left[\right.$ trans $-\mathrm{Ni}(I I) \mathrm{Cl}_{2}$ $\left.\times(o \text {-phenylenediamine })_{2}\right]$.

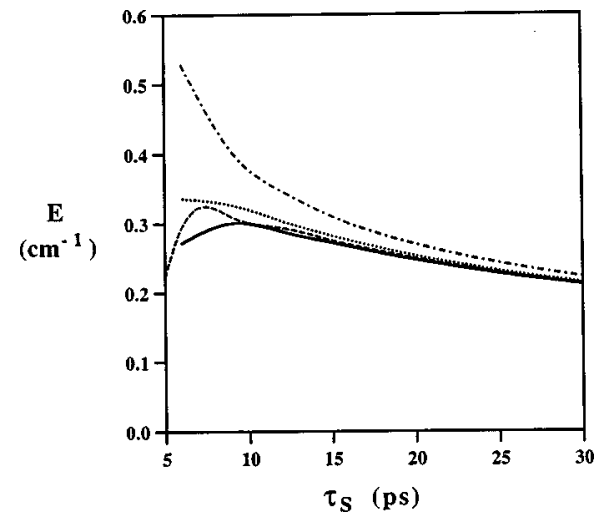

FIG. 5. Values of $E$ and $\tau_{S}$ which acceptably fit the low field relaxation data of $\mathrm{Ni}(I I) \mathrm{Cl}_{2}(o \text {-dpa })_{2}$ in Fig. 3. Values of $D$ are $3 \mathrm{~cm}^{-1}$ (dash-dot); $5 \mathrm{~cm}^{-1}$ (dots); $7 \mathrm{~cm}^{-1}$ (dashes); $10 \mathrm{~cm}^{-1}$ (solid).

$10 \%$ at fields above the low-field limit cannot be excluded. $R_{1 p}$ increased with $B_{o}$ in the range $B_{o}>0.5 \mathrm{~T}$ in a manner that is qualitatively similar to that observed previously for $\left[\mathrm{Ni}(I I)(\mathrm{acac})_{2}\left(\mathrm{H}_{2} \mathrm{O}\right)_{2}\right]$. This behavior supports the hypothesis that phenomena described in the previous section are involved: Namely, that in the low-field limit, the relaxation efficiency is suppressed by a rhombic zfs interaction (a zfs $E$-term) which induces oscillation in $\left\langle S_{\hat{z}}\right\rangle$, thus decoupling the nuclear spin from the dipolar field of the electron spin. When, with increasing $B_{o}$, the Zeeman energy becomes larger than $2 E$, the oscillation of $\left\langle S_{\hat{z}}\right\rangle$, damps out, and $\left\langle S_{\hat{z}}\right\rangle$ develops a static component, which approaches as a limit $m_{S}= \pm 1$ in the uncoupled $|1, \pm 1\rangle$ eigenstates.

We have fit the experimental data using the zfs-limit analytic theory. ${ }^{2}$ Only two fitting parameters were permitted to vary: The zfs parameter $E$ and the electron spin relaxation time $\tau_{S}$, the latter assumed to be independent of magneticfield strength (see below). As described above, the fit is almost independent of the zfs $D$ parameter over the experimental range of field strengths. The reorientational correlation time $\tau_{R}^{(1)}$ was estimated from the Debye equation to be $\sim 430$ ps. Since $\tau_{R}^{(1)} \gg \tau_{S}$, the fits were largely independent of $\tau_{R}^{(1)}$.

The procedure used to generate the fit is the same as that used previously ${ }^{1}$ for $\left[\mathrm{Ni}(I I)(\mathrm{acac})_{2}\left(\mathrm{H}_{2} \mathrm{O}\right)_{2}\right]$. First, the experimental low-field limiting value of $R_{1 p}$ was fit in trial calculations in which both $|E|$ and $\tau_{S}$ were varied. From these calculations, parameter pairs $\left(|E|, \tau_{S}\right)$ which were consistent with the low-field data were obtained and are plotted in Fig. 5. For $\tau_{S} \geqslant 10 \mathrm{ps}$ and $|D| \geqslant 3 \mathrm{~cm}^{-1}$, there was little dependence on the cylindrical zfs parameter $D$. In the second step of the fitting procedure, values of $E$ and $\tau_{S}$ corresponding to points on the curve of Fig. 5 were used in an attempt to reproduce the field dependence of the experimental $R_{1,2 p}$ data. Three such fits, corresponding to $\tau_{S}=9,18$, and $30 \mathrm{ps}$ and $|E|=0.326,0.263$, and $0.213 \mathrm{~cm}^{-1}$, are shown in Fig. 3 . Acceptable fits to the experimental data were found for $\tau_{S}$ $=18 \pm 4 \mathrm{ps},|E|=0.26 \pm 0.03 \mathrm{~cm}^{-1}$.

The analysis supports the central theoretical prediction of the zfs-limit theory described above, namely, that in the low-field limit, the nuclear and electron spins are largely decoupled by an oscillation in $\left\langle S_{\hat{z}}\right\rangle$ that is induced by the rhombic splitting $2 \omega_{E}$. When the Zeeman energy is larger 
than $2 \omega_{E}$, the oscillation is damped, and $\left\langle S_{z}\right\rangle$ develops a large static component. This component couples relatively efficiently to the nuclear-magnetic moment, resulting in the marked increase in $R_{1,2 p}$ which occurs at field strengths above about $B_{o} \approx 0.3 \mathrm{~T}$.

It is interesting that theory also predicts that the ratio $\left(R_{2 p} / R_{1 p}\right) \cong 1.1$ is significantly greater than unity when $B_{o}$ $\approx 1 \mathrm{~T}$, although the experimental intramolecular relaxation data are not sufficiently precise to test this prediction.

\section{Discussion}

Several other high-spin $\mathrm{Ni}(I I)$ complexes exhibit qualitatively similar magnetic-field dependence in the NMR relaxation enhancement as that of Fig. 3. These systems include (1) $\mathrm{Ni}(I I)(\mathrm{acac})_{2}\left(\mathrm{H}_{2} \mathrm{O}\right)_{2}$, which we analyzed previously, ${ }^{1}$ reaching similar conclusions to those drawn here; (2) the $\mathrm{Ni}(I I)$-ethyleneglycol solvate; ${ }^{30}$ (3) a $\mathrm{Ni}(I I)$ substituted form of bovine carbonic anhydrase; ${ }^{31}$ and (4) the $\mathrm{Ni}(I I)$ complex, bis(2,2,6,6-tetramethyl-3,5heptanedionato) $\mathrm{Ni}(I I)$ (aniline- $\left.\left.d_{5}\right)_{2}\right) .{ }^{32}$ Among these studies, only those involving $\mathrm{Ni}(I I)(\mathrm{acac})_{2}\left(\mathrm{H}_{2} \mathrm{O}\right)_{2}$ (Ref. 1) and $\mathrm{Ni}(I I) \mathrm{Cl}_{2}(o \text {-pda })_{2}$ (this work) have been subjected to an analysis which incorporates the effects of zfs rhombicity described above. However, from the similarities of the $T_{1}$ field dispersion profiles, we expect that the physical NMR relaxation mechanisms in all five of these systems are similar.

The functional form of the data does not, per se, uniquely characterize the relaxation mechanism. It is interesting that the characteristic functional form of the field dispersion profiles of these systems (i.e., a pronounced rise in $R_{1 p}$ with increasing field strength which occurs at fields above about $0.5 \mathrm{~T}$ ) can be simulated using simple Zeemanlimit theory by assuming an appropriate $\tau_{v}$ parameter in Eq. (5) to simulate field dependence in $\tau_{S}$. This picture is, of course, unrealistic in that it ignores the effects of zfs-driven spin motions and uses electron spin transition frequencies which are nonphysical both in magnitude and magnetic-field dependence.

In an interesting study of the high-spin $\mathrm{Ni}(I I)$ complex bis(2,2,6,6-tetramethyl-3,5-heptanedionato) Ni(II) $\left.\left(\text { aniline- } d_{5}\right)_{2}\right)\left(=\mathrm{Ni}\right.$-dpm), Larsson et al. ${ }^{32}$ observed $R_{1 p}$ behavior that is qualitatively similar to that in Fig. 3. Their analysis used a model which incorporates a uniaxial zfs tensor $(|D|\rangle 0, E=0)$ which is subject to diffusive reorientational motions plus "pseudorotation"-like fluctuations of the metal coordination sphere. The stochastic motions produce electron spin relaxation. Although their model ignores the effects of zfs-rhombicity, it was capable of simulating the observed rise in $R_{1 p}$ which occurs in the vicinity of $1 \mathrm{~T}$, and good fits to the data were achieved irrespective of the assumed value of $|D|$ over a range $0 \leqslant|D| \leqslant 10 \mathrm{~cm}^{-1}$. While a direct comparison of their results with results of the analytic theories is not straightforward, it appears that the fielddependence results from the processes which produce electron spin relaxation (through the parameters $\tau_{P}$ and $\Delta_{T}$ ) in a way that is similar physically to the field dependence of B-M theory.

Our analysis, in contrast, assumes that the $\tau_{S}^{(\mu)}$ are fieldindependent quantities: Attention is focused on the effects of

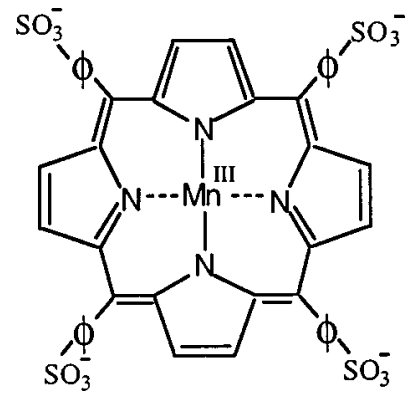

FIG. 6. Structure of $\mathrm{Mn}(I I I)$-tetraphenylporphyrin sulfonate (Mn-TPPS).

the Zeeman field on the spin eigenfunctions and spin dynamics, as described above. We have shown that these phenomena are capable of describing the entire observed magneticfield dependence of the NMR-PRE without the need to assume additional field dependence in $\tau_{S}$. Studies of $\mathrm{Ni}(I I)$ complexes which lack a static rhombic zfs component due to the presence of a threefold or higher molecular symmetry axis would be informative in assessing further the relative contributions of these two mechanisms to the field dependence of the $T_{1}$ field dispersion profile (FDP).

\section{$S=2: \operatorname{Mn}(I I I)$ TETRAPHENYLPORPHYRIN SULFONATE}

$\mathrm{Mn}(I I I)$ porphyrins, such as $\mathrm{Mn}(I I I)$ tetraphenylporphyrin sulfonate (Mn-TPPS, Fig. 6), provide examples of an $S=2$ ion in a site of $D_{4 h}$ symmetry. The rhombic zfs terms vanish so that the $m_{S}= \pm 1$ non-Kramers doublet is unsplit, but a fourth-rank CF term is present which splits the $m_{S}$ $= \pm 2$ non-Kramers doublet. The quadratic cylindrical zfs splitting of $\mathrm{Mn}(I I I)$-TPPS with axial water ligation is ${ }^{33}$ $D=-2.3 \mathrm{~cm}^{-1}$. The fourfold zfs splitting induces an oscillation of $\left\langle S_{\hat{z}}\right\rangle$ within the $m_{S}= \pm 2$ manifold, which, if $\omega_{ \pm 2} \tau_{S} \geqslant 1$, depresses the contribution of the $m_{S}= \pm 2$ doublet to the NMR-PRE. This depression, when present, can be relieved by an applied Zeeman field when the Zeeman energy exceeds the $m_{S}= \pm 2$ doublet splitting. Thus the magnetic-field dispersion profile of $R_{1 p}$ due to $\mathrm{Mn}(I I I)$-TPPS is expected to exhibit a marked rise of $R_{1 p}$ with increasing field strength in the range where the change of spatial quantization occurs within the $m_{S}= \pm 2$ manifold. The splitting of the $m_{S}= \pm 2$ doublet results from a fourth rank $\mathrm{CF}$ component, which we expect to be smaller than typical second rank splittings in orthorhombic complexes. Thus the rise in $R_{1 p}$ is expected to occur at relatively low magnetic-field strengths. The $m_{S}= \pm 1$ manifold is not split by the static CF of Mn-TPPS, and its contribution to $R_{1 p}$ will be approximately field-independent in the low field range.

The solvent proton $T_{1}$ magnetic-field dispersion profiles (FDP) of $\mathrm{Mn}(I I I)$-TPPS at 5, 20, and $35^{\circ} \mathrm{C}$, taken from Ref. 9, are shown in Fig. 7. Because the solvent is in rapid chemical exchange equilibrium with axial sites in the complex, the measured relaxation rate provides the relaxation rate $R_{1 p}$ of the protons of axial water ligands. The qualitative form of the FDP is highly unusual: $R_{1 p}$ is field-independent at very 


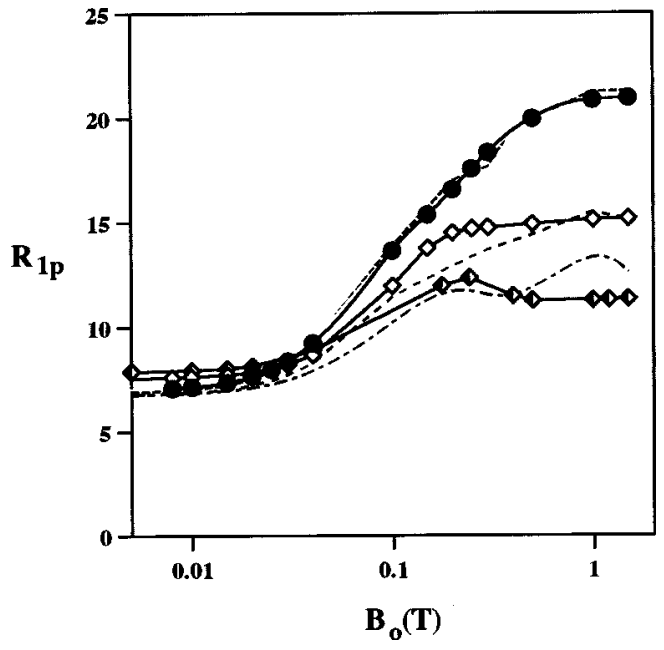

FIG. 7. Simulations of the $R_{\mathrm{I} p}$ magnetic-field dispersion profiles of $\mathrm{Mn}$ TPPS (Ref. 9). Experimental curves are shown by solid lines, with the following symbols for data points: $5^{\circ} \mathrm{C}(\bullet), 20^{\circ} \mathrm{C}(\diamond)$, and $35^{\circ} \mathrm{C}(\diamond)$. Simulated profiles are shown by dots $\left(5^{\circ} \mathrm{C}\right)$, dashes $\left(20^{\circ} \mathrm{C}\right)$, and dot-dash $\left(35^{\circ} \mathrm{C}\right)$.

low-field strengths but rises rapidly at field strengths above about $B_{o}=0.04 \mathrm{~T}$. The rise continues to a field strength in the range $0.2-0.4 \mathrm{~T}$, where a second qualitative change in slope occurs. In the higher field region, $R_{1 p}$ is markedly temperature dependent, the value for $5^{\circ} \mathrm{C}$ being approximately double that for $35^{\circ} \mathrm{C}$. The experimental $R_{1 p}$ profiles have been fit quantitatively by Zeeman-limit (SBM) theory, but Zeeman-limit assumptions are not appropriate to the physical situation, and not surprisingly, the resulting fitting parameters (Table I) are in a number of respects nonphysical (this was recognized by the authors ${ }^{9}$ ). In particular, very short, highly temperature-dependent Mn-proton interspin distances ranging from $2.13 \AA\left(35^{\circ} \mathrm{C}\right)$ to $2.48 \AA\left(5^{\circ} \mathrm{C}\right)$ were

TABLE I. Comparison of fitting parameters for Mn-TPPS obtained from an analysis based on slow motion theory (Ref. 2) (columns 2-4) and Zeemanlimit SBM theory (Ref. 9) (columns 5-7). Omitted values are parameters not used by the theory. The slow motion fit assumed $D=4.0 \mathrm{~cm}^{-1}$, but the fit is nearly independent of this parameter. $\Delta \epsilon( \pm 2)=24 B_{4}^{(4)}$ is the splitting of the $m_{S}= \pm 2$ non-Kramers manifold. The value of $\tau_{R}^{(1)}$ used in the slow motion fits was computed from the value measured (Ref. 37) by C-13 NMR for $\mathrm{Zn}(I I)$-TPP in $\mathrm{CHCl}_{3}$, after correcting for differences in viscosity and temperature with the Debye equation. This estimate provides a lower limit to $\tau_{R}^{(1)}$, since it neglects the effects of peripheral sulfonate groups which undoubtedly slow the reorientation of Mn-TPPS in aqueous solution. The corresponding reorientational parameter in the Zeeman-limit theory is $\tau_{R}^{(2)}$; the values listed are $\tau_{R}^{(1)}=3 \tau_{R}^{(2)}$, where $\tau_{R}^{(2)}$ was determined by the fit to NMR-PRE data (Ref. 9).

\begin{tabular}{|c|c|c|c|c|c|c|}
\hline \multirow[b]{2}{*}{$T$} & \multicolumn{3}{|c|}{ Slow motion theory } & \multicolumn{3}{|c|}{ Zeeman-limit theory } \\
\hline & $5^{\circ} \mathrm{C}$ & $20^{\circ} \mathrm{C}$ & $35^{\circ} \mathrm{C}$ & $5^{\circ} \mathrm{C}$ & $20{ }^{\circ} \mathrm{C}$ & $35^{\circ} \mathrm{C}$ \\
\hline$\tau_{R}^{(1)}(\mathrm{ps})$ & 1400 & 900 & 600 & 690 & 276 & 150 \\
\hline$\tau_{S}^{( \pm 1)}(\mathrm{ps})$ & 456 & 506 & 685 & & & \\
\hline$\tau_{S}^{( \pm 2)}(\mathrm{ps})$ & 261 & 180 & 142 & & & \\
\hline$\tau_{S O}(\mathrm{ps})$ & & & & 25 & 16 & 13 \\
\hline$\tau_{v}(\mathrm{ps})$ & & & & 72 & 47 & 33 \\
\hline $2 \Delta \epsilon_{ \pm 2}^{\circ}\left(\mathrm{cm}^{-1}\right)$ & 0.20 & 0.20 & 0.20 & & & \\
\hline$r_{I S}(\bar{\AA})$ & 2.80 & 2.80 & 2.80 & 2.48 & 2.25 & 2.13 \\
\hline
\end{tabular}

required (in comparison, the distance from $\mathrm{Mn}$ to the water oxygen is $2.24 \AA$ in the x-ray structure ${ }^{34}$ ). Also, the correlation time $\tau_{v}$ for the distortional motions of the zfs tensor, which describes magnetic-field dependence in the electron spin relaxation time, was very long, $\tau_{v} \cong 50 \mathrm{ps,} \mathrm{about} \mathrm{three-}$ fold longer than the low-field electron spin relaxation time, $\tau_{S 0} . \tau_{v}$ is the critical parameter of the Zeeman-limit analysis, since it is the only parameter (within Zeeman-limit theory) which produces a rise in $R_{1 p}$ with increasing field strength. A very long value of $\tau_{v}$ is required to fit the observed rise in the FDP of Mn-TPPS because of the very low-field strength at which this rise occurs, $\sim 0.04 \mathrm{~T}$.

The slow motion theory [Eq. (2)] provides a fundamentally different physical interpretation in which the field dependence of $R_{1 p}$ results principally from field dependence in the spin wave function of the $m_{S}= \pm 2$ levels and the associated changes in the spin motion as described above. An approximate fit of the data has been obtained based on the slow-motion theory [Eq. (2)]. We assume, consistent with the discussion of the preceeding sections, that $R_{1 p} \cong R_{1 \hat{z}}$, and that reorientation is slow enough for Eq. (2) to be valid. The $|0\rangle$ eigenstate does not contribute appreciably to $R_{1 p}$ in the vicinity of the zfs-limit. The $| \pm 1\rangle$ eigenstates contribute an amount equal to

$$
R_{1 p, \pm 1}=C\left\{2\left|\left\langle+1\left|S_{\hat{z}}\right|+1\right\rangle\right|^{2} J(0)\right\},
$$

and the $\left| \pm 2^{\prime}\right\rangle$ eigenstates an amount,

$$
\begin{aligned}
R_{1 p, \pm 2^{\prime}}= & C\left\{2\left(c_{1}^{\prime}\right)^{2}\left|\left\langle+2^{\prime}\left|S_{\hat{z}}\right|+2^{\prime}\right\rangle\right|^{2} J(0)\right. \\
& \left.+2 c_{1}^{\prime} c_{2}^{\prime}\left|\left\langle+2^{\prime}\left|S_{\hat{z}}\right|-2^{\prime}\right\rangle\right|^{2} J\left(\omega_{ \pm 2}\right)\right\}_{s a},
\end{aligned}
$$

where $\quad C=\left(\frac{4}{3}\right)\left(\gamma_{I} g_{e} \beta_{e} r_{I S}^{-3}\right)^{2}\left(\mu_{o} / 4 \pi\right)^{2} 5^{-1}\left[1+P_{2}\left(\cos \theta_{\hat{z}}\right)\right]$. The curly brackets in the second expression indicate a spatial average over the angle $\theta_{\hat{z}}$ between the molecular $\hat{z}$ and laboratory $z$ axes. $R_{1 p, \pm 2}$ in Eq. (11b) must be evaluated by diagonalizing $H_{S}$ at several orientations ( $\left.\theta_{\hat{z}}\right)$ at each specified value of $B_{o}$ and computing the indicated spatial average. This was done in simulations using the symbolic mathematical program Maple. In general, the electron spin relaxation time $\tau_{S}$ may differ for the levels of the $m_{S}= \pm 1$ and \pm 2 spin manifolds, and $\tau_{S}$ may be magnetic field-dependent as well. For simplicity, we assumed that $\tau_{S}$ is field-independent but allowed $\tau_{S}^{( \pm 1)}$ to differ from $\tau_{S}^{( \pm 2)}$. A single, temperatureindependent interspin distance of $r_{I S}=2.80 \AA$, consistent with the measured $\mathrm{Mn}-\mathrm{O}$ bond length of $2.24 \AA$ in Mn-TPPS, ${ }^{29}$ was assumed.

These assumptions produced simulations shown in Fig. 7 and the fitting parameters listed in Table I. The rise of $R_{1 p}$ in the vicinity of $0.04 \mathrm{~T}$ was modeled well by the effects of the changing eigenfunctions $\left|\psi_{ \pm 2}\right\rangle$ without the need to assume magnetic-field dependence in $\tau_{S}$. The fit to the $5{ }^{\circ} \mathrm{C}$ data was quite good, while the fits at $T=20^{\circ} \mathrm{C}$ and $T=35^{\circ} \mathrm{C}$ were somewhat less quantitative. In part this may be due to the limitations of slow motion theory, since $\tau_{S}^{( \pm 1)}$ approaches $\tau_{R}^{(1)}$ at the higher temperatures. Our central purpose here is to understand the physical origin of the field dependence of $R_{1,2 p}$, and more specifically, to investigate whether the changes in the electron spin wave function which occur when the Zeeman energy is comparable to the small intra- 

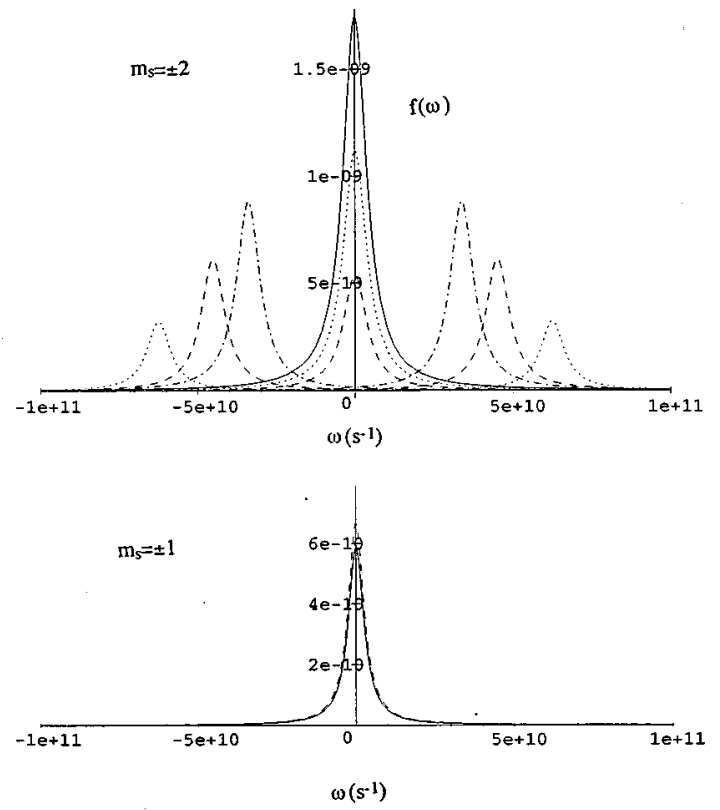

FIG. 8. Dipolar power density produced by the $m_{S}= \pm 2$ eigenstates (top) and $m_{S}= \pm 1$ eigenstates (bottom) of Mn-TPPS. The function plotted on the ordinate is $f(\omega) \equiv\left|\left\langle\mu\left|S_{\hat{r}}\right| \nu\right\rangle\right|^{2} J_{\hat{r}}\left(\omega_{\mu \nu}-\omega\right)$. The values $f(0)$ are the quantities in Eq. (2) of the text. The polar angle between the molecular $\hat{z}$ axis and the laboratory $z$ axis was assumed to be $\theta=\pi / 4$. Curves are shown for $\left(g_{e} \beta_{e} B_{0}\right) /\left(\Delta \varepsilon_{ \pm 1 / 2}\right)=0$ (dot-dash), 0.05 (dash), 0.1 (dots), and 1.0 (solid lines), where $\left(\Delta \varepsilon_{ \pm 1 / 2}\right)$ is the splitting of the $m_{S}= \pm 1 / 2$ manifold.

doublet zfs splittings, as described above, is responsible for the highly unusual low field rise in the FDP of Mn-TPPS. The theory indeed provides a straightforward description of this feature.

\section{Discussion}

The magnetic-field dependence of the relaxation mechanism can conveniently be visualized through plots of the dipolar power density. This is shown in Fig. 8, where the quantity plotted on the ordinate is $f(\omega)$ $\equiv\left|\left\langle\mu\left|S_{\hat{z}}\right| \nu\right\rangle\right|^{2} J_{\hat{z}}\left(\omega_{\mu \nu}-\omega\right)$. The functions $f(0)$ are proportional to the zero-frequency dipolar power produced by the matrix element $\left\langle\mu\left|S_{\hat{z}}\right| \nu\right\rangle$, and thus they describe the NMR relaxation efficiency associated with the various terms of Eq. (2). The functions plotted in Fig. 8 are those corresponding to diagonal and off-diagonal matrix elements within the $m_{S}$ $= \pm 2$ (upper) and $m_{S}= \pm 1$ (lower) manifolds. For the $m_{S}$ $= \pm 1$ levels, only diagonal matrix elements are nonzero, and these are almost field independent within the range of variation of Fig. 8. Since the associated spectral density functions are centered at zero frequency, these terms contribute significantly to the relaxation efficiency. In contrast, the matrix elements within the $m_{S}= \pm 2$ manifold are highly field dependent. Only the off-diagonal matrix elements are nonvanishing in the $B_{o}=0$ limit, but these decrease with increasing $B_{o}$ and are displaced to higher frequency. The off-diagonal matrix elements contribute negligibly to the overall relaxation mechanism. The diagonal matrix elements increase with increasing $B_{o}$, and since these matrix elements describe zero-frequency magnetization, the relaxation efficiency increases accordingly.
Another highly unusual aspect of the Mn-TPPS NMRPRE is the very large molar relaxivity produced by the $\mathrm{Mn}(I I I)$ ion. Although $\mathrm{Mn}(I I I)$ is usually considered a weakly relaxing ion, its proton relaxivity, per mole of bound water, is higher in Mn-TPPS than in hexaqua- $\operatorname{Mn}(I I)$. This undoubtedly reflects an unexpectedly long electron spin relaxation time as was found from the slow-motion theoretical simulations. (The Zeeman-limit fits also required a long $\tau_{S}$, although in this case a long value is achieved by assuming that $\tau_{S}$ is field dependent and by assuming a very long correlation time, $\tau_{v}$, for distortion of the zfs tensor.) The long value of $\tau_{S}$ probably results from the very tight coordination geometry of the $\mathrm{Mn}(I I I)$-porphyrins, specifically from the fact that low-frequency in-plane fluctuations in the zfs tensor, which are needed for electron spin relaxation along the $\hat{z}$ axis, are strongly suppressed in the highly rigid coordination environment of the porphyrin. A detailed analysis of the electron spin relaxation time and its magnetic field dependence in this complex would be interesting.

\section{CONCLUSIONS}

The NMR relaxation enhancements produced by integer spin systems can be influenced strongly by the small zfs splittings (the splittings within the non-Kramers doublets). The CF potential components which produce the intradoublet splittings drive relatively low-frequency oscillations in the time correlation function $\left\langle S_{z}(0) S_{z}(t)\right\rangle$. These motions act to depress the NMR relaxation enhancement in the zfs-limit relative to the enhancement of comparable symmetric complexes in which the noncylindrical zfs terms are nonzero. The presence of a small Zeeman field, comparable in magnitude to the intradoublet spacing but small compared to the large interdoublet zfs splittings, causes a large change in the spin wave functions and has profound effects on the NMRPRE. In the absence of the Zeeman field, the spin eigenfunctions have cartesian polarizations, and only off-diagonal matrix elements of $\left\langle S_{z}\right\rangle$ are nonvanishing. These matrix elements oscillate at the intradoublet splitting and are thus motionally decoupled from the nuclear spin. In the presence of an applied Zeeman field, the diagonal matrix elements of $\left\langle S_{z}\right\rangle$, which are associated with nonoscillating magnetization, grow in magnitude while the oscillating off-diagonal terms decrease. This phenomenon results in an increase of $R_{1 p}$ with increasing field strength which may be quite substantial.

The importance of this mechanism as the origin of the characteristic functional form of the FDP for $S=1$ ions in rhombic site symmetry is supported by analyses of data from two $S=1$ complex ions, $\mathrm{Ni}(I I)(\mathrm{acac})_{2}\left(\mathrm{H}_{2} \mathrm{O}\right)_{2}$ (Ref. 1) and $\mathrm{Ni}(I I)(o \text {-pda })_{2} \mathrm{Cl}_{2}$ (this work), and is consistent with published data for three other systems. ${ }^{30-32}$ An alternate mechanism in which the field dependence of the FDP results from the field dependence of electron spin relaxation may also be important, but the contribution of this mechanism is less clear at present. Data on $S=1$ systems which lack zfs rhombicity would be informative in assessing the relative importance of these two mechanisms.

The $S=2$ spin system, $\operatorname{Mn}(I I I)$-TPPS, exhibits analogous behavior which, as shown above, arises from the effects 
of a static fourth rank splitting of the $m_{S}= \pm 2$ non-Kramers doublet. Five other homologous $\mathrm{Mn}(I I I)$ porphyrins exhibit relaxation behavior which is qualitatively and quantitatively similar to that of $\mathrm{Mn}(I I I)$-TPPS. ${ }^{13}$ Thus we expect generally that the small intradoublet splittings will be of importance for the NMR-PRE properties of integer spin complexes when a substantial static zfs interaction is present and when the Zeeman energy is less than the large zfs splittings ( $D$ and $3 D)$.

There are, of course, specific chemical situations for $S$ $=1$ and $S=2$ where this phenomenon will probably not be important, as, for example, in the $\mathrm{Ni}(I I)$ aquacation, where the static zfs tensor vanishes as a result of octahedral site symmetry of the metal. Also, the presence of a dynamic Jahn-Teller distortion, as occurs in some complexes of $\mathrm{Mn}(I I I)$ which have pseudo-octahedral coordination geometry [such as $\mathrm{Mn}(\mathrm{acac})_{3}$ ], may well suppress the zfs-related relaxation phenomena described above.

\section{EXPERIMENT}

The paramagnetic complex $\mathrm{Ni}(o-\mathrm{pda})_{2} \mathrm{Cl}_{2}$, trans-bis( $o$-phenylenediamine) nickel $(I I)$ dichloride, was synthesized by the procedure of Coakley. ${ }^{35}$ In this method, a $1: 2$ mole ratio of $\mathrm{NiCl}_{2} \cdot 6 \mathrm{H}_{2} \mathrm{O}$ to $o$-phenylenediamine was added to ethanol with stirring. The solution was allowed to reflux for approximately one hour in a hot water bath maintained between $75-80^{\circ} \mathrm{C}$. The light blue precipitate was purified by washing with absolute ethanol and then dried for several hours at $80-85{ }^{\circ} \mathrm{C}$ under vacuum. The $\mathrm{Ni}(I I)$ complex was characterized by IR and magnetic susceptibility measurements, which were in excellent agreement with the literature. ${ }^{36}$ A $40.0 \mathrm{mM}$ solution of $\left[\mathrm{Ni}(o-\mathrm{pda}){ }_{2} \mathrm{Cl}_{2}\right]$ in dimethylsulfoxide (DMSO) was prepared, and a $200 \mu \mathrm{L}$ aliquot transferred to a $10 \mathrm{~mm}$ (ID) glass tube that had been washed overnight in concentrated sulfuric acid to remove labile paramagnetic ions on the surface. The sample was degassed by four freeze-pump-thaw cycles and sealed under vacuum.

Measurements of $T_{1}$ and $T_{2}$ of the $(4,5)$ aromatic protons of $\mathrm{Ni}(I I)(o \text {-dpa })_{2} \mathrm{Cl}_{2}$ were carried out as previously described. ${ }^{1} T_{1}$ measurements used a modification of the inversion-recovery sequence in which pulse triplets are used to sample the magnetization and then to restore it to the $z$ axis. Points of the decay corresponding to times less than about two times the $T_{1}$ of the $(3,6)$ aromatic resonance were ignored in the analysis of the data in order to suppress the contribution of that resonance (see above). $T_{2}$ was measured ${ }^{1}$ using the Carr-Purcell-Meiboom-Gill (CPMG) sequence with a pulse spacing of $1 \mathrm{~ms}$. $R_{1,2 d}$ were also measured for the diamagnetic solvent. Reported values of the paramagnetic part of the relaxation rate, $R_{1,2 p}$, equal the measured relaxation rate minus the relaxation rate of the diamagnetic solvent, which was measured separately. The temperature of the probe was controlled with a stream of dry nitrogen and maintained at $20.0 \pm 0.5^{\circ} \mathrm{C}$.

\section{ACKNOWLEDGMENTS}

This research was supported by the U.S. National Science Foundation in the form of a research grant, CHE-
9423351. S.M.A. received financial support in the form of a Rackham Merit Fellowship from the University of Michigan.

\section{APPENDIX}

The spin functions $O_{q}^{(k)}$ in Eq. (6) are the following:

$$
\begin{aligned}
O_{0}^{(2)}= & 3 S_{z}^{2}-S(S+1), \\
O_{2}^{(2)}= & 2^{-1}\left(S_{+}^{2}+S_{-}^{2}\right)=S_{x}^{2}-S_{y}^{2}, \\
O_{0}^{(4)}= & 35 S_{z}^{4}-30 S(S+1) S_{z}^{2}+25 S_{z}^{2}-6 S(S+1)+3 S^{2}(S \\
& +1)^{2}, \\
O_{2}^{(4)}= & 2^{-1}\left\{\left(7 S_{z}^{2}-S(S+1)-5\right)\left(S_{+}^{2}+S_{-}^{2}\right)\right\}_{S}, \\
O_{4}^{(4)}= & 2^{-1}\left(S_{+}^{4}+S_{-}^{4}\right) .
\end{aligned}
$$

$\{A, B\}_{S}$ is the symmetrized operator, $2^{-1}(A B+B A)$. A more extensive compilation is given in Table 16 of Abragam and Bleaney. $^{28}$

${ }^{1}$ S. M. Abernathy and R. R. Sharp, J. Phys. Chem. 101, 3692 (1997).

${ }^{2}$ R. Sharp, S. M. Abernathy, and L. L. Lohr, J. Chem. Phys. 107, 7620 (1997).

${ }^{3}$ H. Fukui, K. Miura, and H. Matsuda, J. Magn. Reson. 88, 311 (1990).

${ }^{4}$ R. R. Sharp, J. Chem. Phys. 98, 6092 (1993).

${ }^{5}$ J.-M. Bovet and R. R. Sharp, J. Chem. Phys. 99, 18 (1993).

${ }^{6}$ S. M. Abernathy and R. R. Sharp, J. Chem. Phys. 106, 9032 (1997).

${ }^{7}$ R. R. Sharp, J. Chem. Phys. 98, 912 (1993).

${ }^{8}$ R. R. Sharp, J. Chem. Phys. 98, 2507 (1993).

${ }^{9}$ S. H. Koenig, R. D. Brown III, and M. Spiller, Magn. Reson. Med. 4, 252 (1987).

${ }^{10}$ K. E. Kellar and N. Foster, Inorg. Chem. 31, 1353 (1992).

${ }^{11}$ I. Bertini, C. Luchinat, M. Mancini, and G. Spina, J. Magn. Reson. 59, 213 (1984).

${ }^{12}$ I. Bertini, C. Luchinat, and J. Kowalewski, J. Magn. Reson. 62, 235 (1985).

${ }^{13}$ R. R. Sharp, J. Chem. Phys. 93, 6921 (1990).

${ }^{14}$ T. Bayburt and R. R. Sharp, J. Chem. Phys. 92, 5892 (1990).

${ }^{15}$ T. Bayburt and R. R. Sharp, J. Phys. Chem. 97, 4558 (1993).

${ }^{16}$ N. Bloembergen and L. O. Morgan, J. Chem. Phys. 34, 842 (1961).

${ }^{17}$ P. O. Westlund, J. Chem. Phys. 108, 4945 (1998).

${ }^{18}$ R. R. Sharp, J. Magn. Reson. 100, 491 (1992).

${ }^{19}$ I. Bertini, O. Galas, C. Luchinat, and G. Parigi, J. Magn. Reson., Ser. A 113, 151 (1995).

${ }^{20}$ A good review of the Stochastic Liouville Formalism and the Decomposition Approximation is that of P.-O. Westlund, in Dynamics of Solutions and Fluid Mixtures by NMR, edited by J. J. Delpuech (Wiley, New York, 1995), p. 173.

${ }^{21}$ N. Benetis, J. Kowalewski, L. Nordenskiold, H. Wennerstrom, and P.-O. Westlund, Mol. Phys. 48, 329 (1983).

${ }^{22}$ N. Benetis, J. Kowalewski, L. Nordenskiold, H. Wennerstrom, and P.-O. Westlund, J. Magn. Reson. 58, 261 (1984).

${ }^{23}$ P.-O. Westlund, H. Wennerstrom, L. Nordenskiold, J. Kowalewski, and N. Benetis, J. Magn. Reson. 59, 91 (1984).

${ }^{24}$ N. Benetis, J. Kowalewski, L. Nordenskiold, and U. Edlund, J. Magn. Reson. 58, 282 (1984).

${ }^{25}$ S. M. Abernathy and R. R. Sharp, J. Chem. Phys. 106, 9032 (1997).

${ }^{26}$ M. Odelius, C. Ribbing, and J. Kowalewski, J. Chem. Phys. 103, 1800 (1995).

${ }^{27}$ M. Odelius, C. Ribbing, and J. Kowalewski, J. Chem. Phys. 104, 3181 (1996).

${ }^{28}$ A. Abragam and B. Bleaney, Electron Paramagnetic Resonance of Transition Ions (Dover, New York, 1970).

${ }^{29}$ G. Swartz Hall and R. H. Soderberg, Inorg. Chem. 7, 2300 (1968).

${ }^{30}$ L. Banci, I. Bertini, and C. Luchinat, Inorg. Chim. Acta 100, 173 (1985).

${ }^{31}$ L. Banci, I. Bertini, and C. Luchinat, Nuclear and Electron Spin Relaxation (VCH, New York, 1991), pp. 134-135. 
${ }^{32}$ T. Larsson, P.-O. Westlund, J. Kowalewski, and S. H. Koenig, J. Chem. Phys. 101, 1116 (1994).

${ }^{33}$ B. J. Kennedy and K. S. Murray, Inorg. Chem. 24, 1552 (1985).

${ }^{34}$ M. M. Williamson and C. L. Hill, Inorg. Chem. 26, 4155 (1987).
${ }^{35}$ M. P. Coakley, Appl. Spectrosc. 22 (4), 310 (1968).

${ }^{36}$ E. J. Duff, J. Chem. Soc. A 1968, 434.

${ }^{37}$ S. Li, S. L. Swindle, S. K. Smith, R. A. Nieman, A. L. Moore, T. A. Moore, and D. Gust, J. Phys. Chem. 99, 3371 (1995). 